\title{
Observaciones, intuiciones y anécdotas, desde la perspectiva del médico, para una teoría de la historia natural de la continuidad interpersonal. Los colores del tiempo
}

\author{
José Luis Turabián Fernándezª, Benjamín Pérez Francob.
}

\author{
a Especialista en Medicina \\ de Familia y Comunitaria. \\ Centro de Salud "Sta. María \\ de Benquerencia" de Toledo. \\ España. \\ bEspecialista en Medicina \\ de Familia y Comunitaria. \\ Centro de Salud "La Estación" \\ de Talavera de la Reina \\ (Toledo). España.
}

\section{Dirección para} correspondencia:

José Luis Turabián. Calderón de la Barca, 24. 45313 Yepes, Toledo.

Correo electrónico: jturabianf@hotmail.com

Recibido el 4 de abril de 2015. Aceptado para su publicación el 2 de junio de 2015.

\section{RESUMEN}

Se aportan elementos para una teoría de la historia natural de la continuidad interpersonal, desde la perspectiva del médico de familia, analizada en base a la reflexión y el recuerdo de dos médicos respecto a su relación con sus pacientes durante 30 años en que han trabajado en el mismo lugar. Es un viaje por los "colores de la historia natural de la continuidad interpersonal", que cambian al paso del tiempo.

Se observa un escenario de demanda creciente cada vez más trivial, con una falta de comprensión por parte del paciente de lo que constituye una buena calidad científico técnica y una comunicación con el médico inapropiada, con una defensa cognitiva y conductual del médico. Podrían describirse distintas tipologías de continuidad interpersonal, que se combinan a lo largo del tiempo, y que esta podría ser discontinua, y experimentarse como "momentos", presentando efectos positivos y negativos.

Palabras clave. Continuidad de la Atención al Paciente. Atención Primaria de Salud. Relaciones Médico-Paciente. Investigación Cualitativa. Medicina Familiar y Comunitaria.

\section{ABSTRACT}

Observations, insights and anecdotes from the perspective of the physician, for a theory of the natural history of interpersonal continuity. The colors of time.

Elements for a theory of natural history of interpersonal continuity are provided, from the perspective of the family doctor, analyzed on the reflection and remembrance of two doctors regarding their relationship with their patients during 30 years working in the same place. It is a journey through the "colors of the natural history of interpersonal continuity", which change over time.

A scenario of increasingly trivial growing demand is observed, with a lack of understanding by the patient of what constitutes a good scientific and technical quality and an inappropriate communication with the doctor, which causes a cognitive and behavioral defense from the family doctor.

They may describe different types of interpersonal continuity, which combine over time, and that it could be discontinuous, and experienced as "moments", presenting positive and negative effects.

Key words. Continuity of Patient Care. Primary Health Care. Physician-Patient Relations. Qualitative Research. Family Medicine.

"La primavera ha crecido con tanta sequedad en esta ciudad, que ahora aprisiona el verano. Hubo una época de adolescencia en que la luz verde teñía las paredes secas y cubiertas con yeso; entonces, en la ciudad parecía que la fresca vegetación congelaba en niebla el humo de las casas. Luego palideció, perdió todos los colores...: un tinte de seco ópalo un poco eclipsado por los siglos. Solo cuando alguno de los colores amarillos o verdes grises discrepan del yeso rojo, el verano detiene su vuelta de forma violenta".

Santucci A. Il colore del tempo. Muri, intonaci e mattoni della Bologna antica e di quella presente. 


\section{INTRODUCCIÓN}

La continuidad de la atención es considerada como una característica definitoria de la Medicina de Familia (MF) y la Atención Primaria (AP) ${ }^{1-3}$. Sin embargo, a pesar de décadas de estudio, permanece difícil de definir y cuantificar ${ }^{4}$, puede verse desde distintas disciplinas ${ }^{5}$ y diferentes perspectivas $^{6}$, siendo motivo de controversia ${ }^{7}$, sugiriendo una jerarquía de dimensiones de menor a mayor complejidad ${ }^{7}$ (tabla 1) y que puede también variar según las diferentes trayectorias de las enfermedades, la presencia o no de multimorbilidad, los momentos evolutivos del episodio de enfermedad, los mecanismos de transferencia y contra-transferencia, la clase social, el género, la raza, la edad, la ideología, los mecanismos de control social y los distintos tipos de sistemas sanitarios y de asistencia ${ }^{8,9}$. Además, parece caracterizarse la relación médicopaciente centrándose en el encuentro episódico de enfermedad ${ }^{10}$.

\section{Dimensiones de la continuidad de atención según grados de complejidad}

Continuidad informativa

Continuidad geográfica

Continuidad interdisciplinaria o en equipo

Continuidad flexible

Continuidad longitudinal o cronológica

Continuidad interpersonal en la relación médico-paciente

Continuidad familiar

\section{Concepto}

Es la disponibilidad de información clínica a cualquier proveedor sanitario que atienda al paciente.

Se refiere a la atención que se proporciona con la continuidad, independientemente de la ubicación del paciente -consultorio, casa, hospital, etc.

Implica el cuidado que permite el conocimiento previo del paciente incluso cuando requiere una amplia gama de servicios especializados.

Es la flexibilidad del servicio en respuesta a las necesidades o situaciones cambiantes.

Se refiere a que el cuidado de la salud se produce en el mismo lugar, con el mismo registro médico, y con los mismos profesionales, de modo que existe un conocimiento creciente del paciente por los que le proporcionan la asistencia; la continuidad longitudinal implica un cierto patrón de visitas, pero no aborda directamente la naturaleza de la relación entre el paciente y el proveedor.

Se refiere a un tipo especial de la continuidad longitudinal en la que hay una relación personal continua entre el paciente y la asistencia, y se caracteriza por la confianza y la responsabilidad personal.

Se refiere a un sistema de atención en el que todos los miembros de la familia reciben asistencia de proveedores -principalmente el médico de familia- que tienen conocimiento de los problemas de salud de otros miembros de la familia, y en la que hay una relación personal continua con todos los miembros de la unidad familiar.

Tabla 1. Jerarquía de dimensiones de la continuidad de atención. Fuente: Elaboración propia

El término "relación médico-paciente" es hoy en día una denominación poco precisa, y debería hablarse preferiblemente de "interacción entre actores de la atención sanitaria”. En consecuencia, se sugiere que el paradigma de la relación médico-paciente debe ser revisado, a tenor de, entre otros factores, la explosión de información médica, las demandas crecientes, la responsabilidad sobre terapias y resultados, el incremento de la complejidad, el aumento de "nuevos" diagnósticos y fármacos, la aparición de la medicina defensiva, el mayor estrés que debe soportar el médico, y la creciente desconfianza de los pacientes, etc ${ }^{11}$.

Todo este escenario lleva a la existencia de una gran diversidad de técnicas de medición para estudiar la continuidad, muchas de las cuales se refieren a patrones y concentración de consultas 
con el mismo proveedor, en lugar de la naturaleza de la continuidad interpersonal $(\mathrm{Cl})^{7,12-14}$. Aunque poco se conoce de los factores que facilitan la continuidad, se describen numerosos beneficios -sin saber exactamente por qué se producen- y se estima que, a lo largo del tiempo, la confianza del paciente mejora, y con ella la calidad de la información, pero sin definir cuánto tiempo de relación médico-paciente es preciso ${ }^{7,9,15-22}$. Sin embargo, la $\mathrm{Cl}$ puede tener también efectos negativos o problemáticos (tabla 2), y hay una tensión entre los esfuerzos dirigidos a mejorar la facilidad de acceso a la asistencia y los dirigidos a fortalecer la $\mathrm{Cl}^{23}$.

\section{Efectos positivos o útiles de la continuidad interpersonal}

Construye confianza y mejora la relación médicopaciente.

Tiene ventajas clínicas: al aumentar el conocimiento sobre el paciente y facilita el diagnóstico y el tratamiento.

Presenta la oportunidad única para poder estudiar la historia natural de la enfermedad.

Permite ver el que la presencia de un problema en un miembro de la familia puede ser un marcador de conflicto en otro u otros miembros de la misma.

Facilita el seguimiento de los enfermos crónicos.

Facilita la implementación de elementos preventivos.

La continuidad tiene impacto en salud pública, pues permite transformar las acciones sobre poblaciones en acciones sobre personas, familias y comunidades.

\section{Colabora a la calidad de la asistencia.}

Favorece la satisfacción con el servicio.

Mejora el cumplimiento terapéutico.

Ahorra costes al sistema sanitario (mejor uso de los servicios, menos hospitalizaciones, etc.).

\section{Efectos negativos o problemáticos de la continuidad interpersonal}

Es difícil, compleja y consume mucha energía.

Puede ser condicionada por la clase social, el género, la raza, la edad, la ideología y los mecanismos de control social, y los distintos tipos de sistemas sanitarios y de asistencia.

El médico no es el único que brinda atención a la salud de los individuos, también está la enfermera, el odontólogo, el psicólogo clínico y otros más.

Los instrumentos para valorarla son de aplicabilidad limitada.

Las medidas de la experiencia del paciente respecto a la continuidad interpersonal pueden variar ampliamente entre pacientes con el mismo médico, y se conoce poco sobre la fuerza de la relación.

Produce un proceso de esclerotización e involución o acostumbramiento que facilita errores diagnósticos.

No sabemos si los teóricos beneficios son exclusivos de ella.

Es confuso el grado en que la calidad de la relación médico-paciente depende de la continuidad longitudinal.

Cabe el peligro de maximizar la continuidad interpersonal menoscabando la "buena práctica".

La relación médico-paciente no es fundamentalmente recíproca y contiene elementos que generan conflicto.

Hay una a tensión entre los esfuerzos dirigidos a mejorar la facilidad de acceso a la asistencia y los dirigidos a fortalecer la continuidad interpersonal.

Tabla 2. Efectos positivos y negativos de la continuidad interpersonal. Fuente: Elaboración propia 
En todo este contexto, se ha puesto menos interés en describir, reflexionar y conceptualizar las posibles variaciones a lo largo del tiempo de la relación de un médico con los pacientes-familias ${ }^{23}$. Así, nos proponemos aportar, mediante la reflexión y el recuerdo -un método cualitativo-, algunos elementos para comenzar a construir una teoría de historia natural de la $\mathrm{Cl}$, a partir de observaciones, intuiciones, anécdotas personales y preguntas sin clara contestación, de las vivencias experimentadas en el devenir de la $\mathrm{Cl}$ de los autores, médicos de familia que llevan atendiendo pacientes en un mismo lugar durante un largo período de tiempo (30 años).

\section{LA HISTORIA NATURAL: "LAS HOJAS PIERDEN SU VIDA VERDE EN OTOÑO, PERO FLORECEN EN COLORES MARAVILLOSOS" ${ }^{24}$.}

En Medicina, un objetivo prioritario debe ser el conocimiento de la historia natural de la enfermedad ${ }^{25}$. Para captar mejor este proceso es interesante prestar más atención a la dimensión humana del científico. La MF presenta la oportunidad única para estudiar la historia natural de la enfermedad, pero también la historia natural de la $\mathrm{Cl}^{26}$. Atender continuadamente al mismo paciente durante treinta años puede ser fascinante ${ }^{27}$. La relación médico-paciente exige que el médico asuma la estructura de sus valores que le impiden ver las cosas desde la perspectiva del enfermo, y así encontramos similitudes entre la historia natural de enfermedad y lo que podríamos considerar diferentes cursos evolutivos de la $\mathrm{Cl}$ (tabla 3) ${ }^{28,29}$.

Como los cambios de los colores de las piedras de la ciudad de Bolonia a lo largo del tiempo ${ }^{30}$ o los colores de las pinturas de la Catedral de Ruen

\begin{tabular}{|c|c|c|}
\hline $\begin{array}{l}\text { Cursos evolutivos de la con- } \\
\text { tinuidad interpersonal* }\end{array}$ & Concepto & Observaciones, intuiciones y anécdotas \\
\hline Simple continua & $\begin{array}{l}\text { Curso evolutivo completo en } \\
\text { sí misma de la continuidad } \\
\text { interpersonal. }\end{array}$ & $\begin{array}{l}\text { El paciente tiene un solo médico de cabecera } \\
\text { toda su vida. }\end{array}$ \\
\hline Crisis aguda o explosiva & $\begin{array}{l}\text { Aparición o desaparición brusca } \\
\text { de la confianza con ruptura o } \\
\text { fortalecimiento de la relación. }\end{array}$ & $\begin{array}{l}\text { Ante una discrepancia o un acuerdo } \\
\text { aparentemente trivial, el paciente se cambia } \\
\text { de médico o expresa una fuerza de la relación } \\
\text { médico-paciente inesperada. }\end{array}$ \\
\hline Lentamente progresiva & $\begin{array}{l}\text { Aparición o desaparición } \\
\text { lentamente progresiva de } \\
\text { la confianza con ruptura o } \\
\text { fortalecimiento de la relación. }\end{array}$ & $\begin{array}{l}\text { Reiteradas visitas muestran progresiva } \\
\text { desconfianza o desacuerdo del paciente con } \\
\text { el médico: "Ya no me atiende Usted como } \\
\text { antes...". }\end{array}$ \\
\hline Paroxística o discontinua & $\begin{array}{l}\text { Se repite con intervalos abruptos } \\
\text { predecibles o no. }\end{array}$ & $\begin{array}{l}\text { Una familia que había atendido durante } \\
\text { muchos años, y cambió de médico por una } \\
\text { discrepancia, regresó al cabo de } 1 \text { año. }\end{array}$ \\
\hline Metastásica & $\begin{array}{l}\text { Se extiende (o puede exten- } \\
\text { derse) espontáneamente a } \\
\text { proveedores diferentes, y en } \\
\text { general distantes, del habitual. }\end{array}$ & $\begin{array}{l}\text { Pacientes que establecen largos períodos de } \\
\text { continuidad con los especialistas secundarios } \\
\text { saltándose al médico de familia. }\end{array}$ \\
\hline Imaginaria o virtual o informal & $\begin{array}{l}\text { Las consultas son siempre } \\
\text { realizadas por un tercero. }\end{array}$ & Siempre consulta un familiar o cuidador. \\
\hline Referida & $\begin{array}{l}\text { El paciente tiene otro médico } \\
\text { de cabecera, pero él mismo o } \\
\text { por mediación de un tercero, } \\
\text { mantiene informado al médico y } \\
\text { le pide consejo. }\end{array}$ & $\begin{array}{l}\text { El paciente o un familiar -del que no soy su } \\
\text { médico- cada vez que le veo (en la sala de } \\
\text { espera, o en la calle) me consulta. }\end{array}$ \\
\hline Insidiosa & $\begin{array}{l}\text { El paciente mantiene } \\
\text { continuamente un médico de } \\
\text { cabecera, pero consulta a otros. }\end{array}$ & $\begin{array}{l}\text { El paciente consulta siempre al médico } \\
\text { más cercano de su trabajo o lugar de estu- } \\
\text { dios, aunque no es su médico de cabecera } \\
\text { formalmente. }\end{array}$ \\
\hline
\end{tabular}

Tabla 3. Tipologías de la historia natural de la continuidad interpersonal. * Los cursos evolutivos pueden combinarse. Fuente: Elaboración propia. 
de Monet $^{31}$, al presentar unas observaciones, intuiciones y anécdotas personales de los cambios causados por el paso del tiempo en la $\mathrm{Cl}$, con una casi ausencia de la perspectiva del paciente, hace que el auténtico protagonista sea el tiempo y el médico que da vida a esa relación con sus pacientes. Se ha comparado la consulta en MF con una película, en la que el episodio de enfermedad es una parte de la totalidad ${ }^{32}$. También encontramos una "película", con sus escenas parciales de cada estadio de la evolución de la $\mathrm{Cl}$, aunque esta no puede reducirse al esquema de una simple curva de ascenso, culminación y declive, sino que hay que tener conciencia de que el juicio es arbitrario, así como decir que el rosal alcanza su plenitud al dar la flor y el peral cuando da sus frutos. Nosotros percibimos (tabla 4) que el médico joven, con su especialidad recién acabada, enfatiza su papel de técnico biomédico ${ }^{10,33,34}$. Es una relación paternalista y la comunicación se sustituye por el diagnóstico ${ }^{9,35}$. Los médicos en España mantienen frecuentemente este estilo de consulta ${ }^{10}$ (¿están en una fase "adolescente" respecto a la continuidad interpersonal?). Al paso del tiempo, el médico ya "no tan joven" puede desarrollar un modelo de Cl de un "Santo", con absolutos de perfección. Estos profesionales son "médicos a quienes era normal que Ilamaran a cualquier hora del día, especialmente de noche..., con más de cien personas al día..., nunca cerraban las puertas y donde la cola se prolongaba hasta la calle..., parte de las visitas que hacía no las cobraba..." 34 .

Puede suceder también que este médico "no tan joven" tome conciencia de que solo con la intervención biomédica y la bondad no puede abordar y resolver los problemas que le presentan los pacientes; así se puede acercar al concepto de participación, educación, comunidad y empatía. Pero la empatía puede estar sobrevalorada ${ }^{36}$ y tal vez no sea cierta la idea de que cuanto más desarrollamos esta capacidad más fuertes y saludables son nuestras relaciones personales. Esta podría quedarse en una fase "romántica" en el caso de que no se desarrollara equilibradamente la asertividad con la empatía. La relación interpersonal es aquella que se establece entre dos seres humanos cuando se tratan mutuamente como personas. Pero, el médico, para ser buen médico, tiene necesariamente que "convertir, en parte, en objeto contemplable el cuerpo del enfermo", sin olvidar que ese cuerpo es una parte más de la persona $^{9}$.

Al paso del tiempo, el médico maduro ve que no todos los pacientes desean participar ni "capacitarse", sino que piden depender del servicio sanitario ${ }^{37}$; también observa cómo un número importante de pacientes tratan de manipularle, son exigentes y maleducados. Recordemos que los signos directos de solicitud de participación pertenecen en exclusiva a una clase psicosociocultural y/o económicamente privilegiada pero los indirectos los muestra toda la población aunque mediante conductas inmaduras. Puede comenzar así una relación con sus pacientes que enfatiza la asertividad. El médico "viejo" puede buscar un replanteamiento de los términos del contrato moral médico-paciente; pide una revisión del pacto existente entre médico y paciente. Sueña con un día en que la caída del hechizo del médico o medicina "best-seller" -del especialista secundario y la tecnología, incluida la farmacológica, así como de la propaganda de los medios de comunicacióndé paso a la reaparición del paciente con talento; evitando "culpar a la víctima" espera un paciente activo y suficientemente abierto que permita una conciencia radicalmente distinta a la suya propia la del MF con su Cl de adulto-adulto. Recordemos que los médicos tienen los mismos marcos determinantes que los pacientes y, por eso, "el error individual" no existiría en la Cl. Una "buena consulta" no supone eliminar las diferencias entre ambas partes, sino que se alcanza un acuerdo temporal sobre el qué hacer (aunque no haya un consenso cultural sobre el "por qué" hacerlo) ${ }^{38}$.

\section{EL SOL DEL MEMBRILLO. ESCENAS DE CONFLICTOS, RIESGOS, CRISIS Y RUPTURAS. ¿EXISTE AÚN LA CONTINUIDAD INTERPERSONAL?}

La película El sol del membrillo (Víctor Erice; 1992) ${ }^{39}$ muestra un estudio sobre los cambios de la luz en los diferentes momentos del día (el paso del tiempo), en que el pintor Antonio López García se encuentra pintando un membrillero. Del mismo modo, cambia la luz y pasa el tiempo en nuestra consulta. Llegados a cierta edad empezamos a ver desaparecer a algunos de nuestros pacientes. Y no solo porque se mueran (tablas 5 y 6 ). Son los que, habiendo sido durante un largo o breve período pacientes aparentemente satisfechos, se esfuman en un limbo remoto. Esta situación se suele vivir con tristeza y preocupación. La causa más frecuente por las que los pacientes cambian de médico es una relación deteriorada entre ambos ${ }^{40}$. Puede haber pacientes con necesidades especiales más problemáticas y la presencia del médico residente o la existencia de un tándem médico-enfermero 


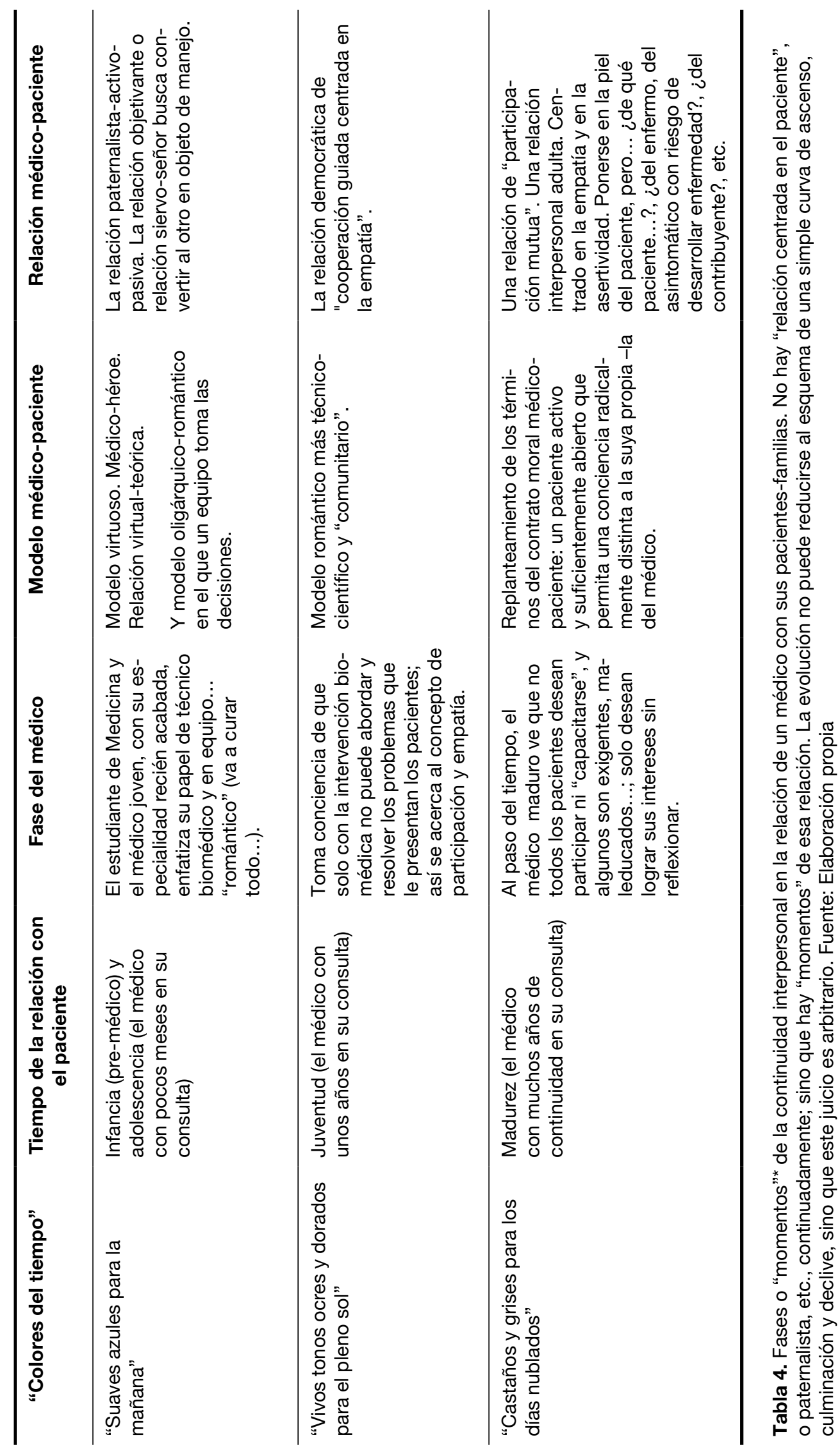




\begin{tabular}{|c|c|}
\hline $\begin{array}{l}\text { Motivos de ruptura de la } \\
\text { continuidad interpersonal }\end{array}$ & $\begin{array}{l}\text { Situaciones que modifican, dificultan o impiden la continuidad } \\
\text { interpersonal y favorecen cambio de médico }\end{array}$ \\
\hline Desavenencia o desacuerdo & $\begin{array}{l}\text { Trastornos psiquiátricos (Somatizaciones-Ansiedad, Psicosis) y/o dependencia a } \\
\text { psicofármacos u opiáceos }\end{array}$ \\
\hline Desconfianza & $\begin{array}{l}\text { Múltiples visitas de los miembros de la familia a diferentes proveedores o un nuevo } \\
\text { diagnóstico grave. }\end{array}$ \\
\hline Alejamiento & $\begin{array}{l}\text { Circunstancias de la consulta: cambio de lugar de residencia; gran demanda y } \\
\text { masificación de consulta; gran lista (“cupo") de pacientes; incompatibilidad de } \\
\text { horarios; la presencia del médico residente; el hecho de presentarse sin posibilidad } \\
\text { de separación la pareja médico-enfermero. }\end{array}$ \\
\hline Confusión emocional & Conflicto derivado de la prescripción (especialmente la inducida). \\
\hline Cambio de gustos & $\begin{array}{l}\text { La creencia por parte del médico de que el paciente atendido durante muchos años } \\
\text { "debe de estar agradecido". }\end{array}$ \\
\hline Períodos que se "cierran" & iento. \\
\hline
\end{tabular}

Tabla 5. Motivos de dificultad y ruptura de la continuidad interpersonal. Fuente: Elaboración propia.

\section{Categorías}

Pasar el umbral de conocimiento "intimo"

Evolución en crisis aguda

\section{Citas textuales-anécdotas}

Una paciente, que había sido visitada en la consulta durante 7 años, contó en una visita, tras un largo silencio, que "fue violada por su abuelo cuando era niña". Después de esa visita nunca más habló del tema en las sucesivas consultas. Al cabo de unos meses se cambió de médico.

Después de 20 años de asistencia, aparentemente con una continuidad deseada, pues vive en una zona que no depende teóricamente del centro de salud, solicita un antibiótico prescrito por dentista particular, que no está indicado. Se le explica.

"Esto no se quedará así. Me cambiaré inmediatamente de médico".

Desconfianza

Una paciente atendida durante más de 10 años, que acude por un problema menor, acompañada por su marido: "Hoy no ha querido atendernos..., me vio su ayudante... [El médico residente]. ¡Ya no quiere nada con nosotros...!".

Desavenencia Al paciente, atendido durante más de 15 años, otro médico le dio un informe, donde automáticamente, con un clic, se imprimieron completamente sus antecedentes personales, que no son depurados a tenor de lo que convenga en esa situación. Ahora acude a consulta enfadado:

-Quíteme del volante eso que pone... [Factores psicosociales, como un intento autolítico, una interrupción voluntaria de embarazo, presencia de problemas económicos, ansiedad, depresión, problemas de relación familiar...].

- ¡Usted la tiene tomada conmigo! Me reservo acudir donde convenga por divulgar esta información privada".

Evolución lentamente progresiva
Mujer diabética, atendida ella y su marido y 3 hijos, desde 15 años en la consulta, a la se le ha cambiado irbesartan que prescribía el endocrinólogo por enalapril y trae ahora informe del especialista: "Su médico cambia irbesartan por enalapril sin saber por qué. Se ruega seguir recetando irbesartan". La paciente solicita la receta.

-“Al final tienes que ver dónde tienes confianza"-le digo.

-"No es cosa de confianza..., es que el endocrino es el que me trata" -contesta ella. 


\begin{tabular}{|c|c|}
\hline $\begin{array}{l}\text { Evolución simple } \\
\text { continua }\end{array}$ & $\begin{array}{l}\text { Una mujer de } 83 \text { años atendida desde hace más de } 20 \text { años. } \\
\text {-"Lo seguiré trayendo mientras pueda..." (un queso manchego por Navidad) [Acaba de fallecer } \\
\text { su marido de } 85 \text { años]. }\end{array}$ \\
\hline Cambio de gustos & $\begin{array}{l}\text {-"Yo no conozco a otro médico de cabecera..., siempre he estado con usted..., ya son veinte } \\
\text { años..., pero..." (A los pocos meses de cambió de médico) }\end{array}$ \\
\hline \multirow[t]{2}{*}{$\begin{array}{l}\text { Períodos que se } \\
\text { "cierran" }\end{array}$} & $\begin{array}{l}\text { "En los últimos 10-15 años he tendido a tutear a los pacientes..., en los últimos años tiendo a } \\
\text { llamarlos nuevamente de usted". }\end{array}$ \\
\hline & $\begin{array}{l}\text { "Desde hace } 15 \text { años suelo dar la mano a cada paciente, pero teniendo en cuenta ese tiempo } \\
\text { y la frecuentación en nuestro contexto, ahora me pregunto si no será adecuado evitar tantos } \\
\text { apretones de mano (aunque sé que el mero contacto cariñoso de cualquier estilo, puede mejo- } \\
\text { rar la salud)". }\end{array}$ \\
\hline \multirow[t]{4}{*}{ Alejamiento } & La consulta para el enfermo ya no es una situación excepcional: \\
\hline & -"Ha pedido cita también tu hija; la tiene luego a las 12,15 horas, pero si la puedo ayudar ya...". \\
\hline & -”Ah! No sé. No me ha dicho nada..., no sabía que venía..." \\
\hline & $\begin{array}{l}\text { Ir al médico no supone ya un acontecimiento de cierta importancia; es tan común como com- } \\
\text { prar el pan..., por eso, del mismo modo que no se avisa a la familia que se va a comprar pan, } \\
\text { no se avisa de que se va al médico. }\end{array}$ \\
\hline $\begin{array}{l}\text { Ruptura de la con- } \\
\text { tinuidad metas- } \\
\text { tásica }\end{array}$ & -"El cardiólogo me ha dado el alta..., ¡después de 10 años! ¡iMe ha matado!!”. \\
\hline Desacuerdo & $\begin{array}{l}\text { El paciente, que es visitado desde hace } 20 \text { años, lleva } 5 \text { meses en situación de Incapacidad } \\
\text { Transitoria tras una pancreatitis aguda atendida en un hospital privado; se le solicita informe. } \\
\text { Sin "despedirse" se cambia de médico. }\end{array}$ \\
\hline $\begin{array}{l}\text { Evolución discon- } \\
\text { tinua }\end{array}$ & $\begin{array}{l}\text { Un varón de } 83 \text { años, con cardiopatía coronaria y disfunción sexual tras cirugía de próstata, tras } \\
15 \text { años de ser atendido en la consulta se cambió de médico al negársele la prescripción de } \\
\text { sildenafilo que solicitaba. Un año después regresa a su antiguo médico. }\end{array}$ \\
\hline
\end{tabular}

Tabla 6. Citas textuales-anécdotas de algunas de las categorías definidas tras el recuerdo y la reflexión sobre tipologías de la historia natural, motivos de dificultad y de ruptura de la continuidad interpersonal. Fuente: Elaboración propia

indivisible, pueden suponer dificultades ${ }^{41,42}$.

Quizás los gustos cambian y alteran las sendas de un destino... Hay determinados períodos destinados a "cerrarse" en cierto momento. Estas rupturas pueden ser importantes en el médico para evitar verse envuelto en un proceso de acostumbramiento. Los pacientes en los que se producen relaciones más profundas con el MF -por ejemplo, porque cuentan temas íntimos- pueden tender a dejar a ese médico o, por el contrario, hacer que la relación sea más fuerte. Por una parte, el tener un médico de referencia, puede implicar un potente factor de protección para circular por el sistema sanitario. Pero, por otra, que el mismo médico deba atender al paciente "desde la cuna a la tumba", es un paradigma que deberíamos cuestionarnos en AP/MF por el aumento de la carga de trabajo, de la complejidad de la multimorbilidad y de las expectativas ${ }^{41}$. Antiguamente los pacientes raramente se cambiaban de médico... ¿lo hacen en los últimos tiempos con más frecuencia? ${ }^{43}$ Es posible que sí. Está claro que hoy la sociedad es más exigente. En nuestra experiencia, la razón más habitual en los últimos años es el no obtener lo solicitado o exigido (receta, prueba complementaria, baja laboral) esté o no indicado, sea o no explicado cuidadosamente, exista o no $\mathrm{Cl}$ con aparente confianza. Son, por tanto, muy frecuentes los problemas relacionados con las transiciones de asistencia ${ }^{44}$. Además, en nuestro contexto se aprecia un fenómeno de los últimos años: el vaciado de los contenidos del MF: las tareas técnicas han perdido sus contenidos conceptuales 
e ideológicos; el verdadero contenido de la tarea del MF ha devenido en la prescripción inducida ${ }^{45}$. El bajo valor que la sociedad concede a la AP afecta negativamente a la $\mathrm{Cl}$.

Frecuentemente se resalta la curiosidad de que la incompetencia técnica del profesional no ocupe un lugar prioritario en esta lista de causas de cambio de médico ${ }^{46}$. No está claro si los pacientes dan más valor a la calidad humana, pero lo que parece existir en los últimos años es un mayor nivel de exigencia, aún a costa de perder la $\mathrm{Cl}$. Por diferentes razones ${ }^{23}$, los MF atienden cada vez más a pacientes no habituales, y el hecho de que esto no origine "cataclismos" en diversos países, sugiere que la $\mathrm{Cl}$ podría no ser tan esencial ni específica de la MF (aunque sea deseable y no haya pasado del todo de moda) ${ }^{47}$. La Cl también supone riesgos. Por una parte, el de errores diagnósticos: el MF puede suponer que ya conoce todo lo significativo del paciente y no realizar investigaciones importantes. Así, los pacientes pueden sentirse mejor con un nuevo médico que sí realiza pruebas complementarias y prescribe los fármacos que no efectuaba el habitual. Aunque probablemente es preferible tener este sesgo que el del médico que siempre empieza de cero con los pacientes que ve. Por otra parte, maximizar la $\mathrm{Cl}$ menoscabando la "buena práctica", dando prioridad a la $\mathrm{Cl}$ "a cualquier precio" (aceptando como norma demandas inadecuadas o decisiones inapropiadas, por ejemplo), llevan a la pérdida del rol de médico y su producción de "sanador" o "cuidador", y fácilmente se cae en la manipulación y la yatrogenia. Meng-tse dijo: "aceptar irreflexivamente lo que tras un maduro examen puede resultar inaceptable sería obrar con ligereza. Conceder cuanto se nos pide, sin reflexionar sobre la conveniencia de tal concesión, no sería más que una manifestación de nuestra debilidad de carácter, y no un acto de generosidad" ${ }^{\prime 8}$. Siempre debería intentarse el equilibrio.

No están claras las características de los pacientes insatisfechos con la comunicación, ni que esta pueda mejorar con mayor tiempo de consulta; la forma en que se usa el tiempo parece ser más importante que la duración total ${ }^{49}$. Tal vez, la historia natural de la $\mathrm{Cl}$ puede equipararse con la relación terapeuta-grupo terapéutico en psicoterapia: dura un determinado tiempo. El inminente fin del grupo terapéutico impulsa a los integrantes a abordar sus problemas vitales con mayor ahínco. Dicho de otra forma, la única manera de que uno pueda salvar su matrimonio es estar dispuesto a dejarlo (y ser capaz de hacerlo). Los mundos separados de la experiencia y referencia (roles) de la persona ordinaria y del profesional son siempre elementos potenciales de conflictos. Los MF suelen encontrar muy frustrantes las consultas por motivos triviales que son innecesarias, y la falta de confianza, respeto, o acuerdo de los pacientes ${ }^{50,51}$. Los cambios en los últimos 20-30 años han hecho a la $\mathrm{Cl}$ actual más problemática por diferentes causas sociales, políticas, morales, psicológicas y técnicas ${ }^{22}$, relacionadas con una trivialización de la sociedad en su conjunto, y están distorsionando por completo la relación médico-paciente en sus aspectos fundamentales, a tal punto que algunos autores plantean que ya no es buena ni mala, sino que simplemente ha dejado de existir. ¿Se puede, entonces, pensar que la historia natural de la $\mathrm{Cl}$ es totalmente dependiente del contexto? No lo creemos; los cambios del contexto probablemente no han hecho sino exacerbar una historia natural consustancial, aunque evidentemente la evolución de la $\mathrm{Cl}$ puede variar de una cultura o contexto a otro $^{51}$.

\section{CONCLUSIONES}

La Cl no es un fin en sí misma, sino una herramienta. Entendemos que la $\mathrm{Cl}$ debe ser más reequilibrada por una "atención centrada en el médico". Lo expuesto podrá parecer fruto de observaciones limitadas. Usamos la generalización y la intuición. Desde luego, un grave sesgo sería el de generalizar partiendo de un hecho aislado. Pero, una virtud es la intuición sabia de lo que puede haber detrás de ese hecho aislado. $Y$ junto al dato identificador $o$ puntualmente representativo, tratamos de mostrar la anécdota reveladora; elegir la anécdota precisa puede confirmar una observación o una teoría. Pero, ¿cómo ser objetivo en observaciones, intuiciones y anécdotas subjetivas? Reflexionando $\mathrm{y}$ argumentando.

James Mackenzie ${ }^{26}$ realizó gran parte de su trabajo original basándose en la observación de individuos durante años. La acumulación de conocimiento sobre la enfermedad puede obtenerse en MF, pero requiere un esfuerzo de observación y de buenos registros. Lo mismo podríamos decir del estudio de la Cl. Aquí, los autores no han llevado un diario durante los últimos 30 años de actividad asistencial, aunque hubiera podido ser una buena base de datos, un instrumento adecuado para estimar efectos, como "el calendario de señor Pere Comas del pueblecito catalán de Cardedeu, treinta kilómetros al norte de Barcelona, donde 
durante más de cincuenta años, desde 1952, un mismo observador ha anotado cuidadosamente las fechas de aparición y caída de las hojas, salida de las flores y de maduración de los frutos de más de cien especies de plantas cultivadas y silvestres. También anotaba las primeras fechas de cada año en que veía golondrinas, vencejos y distintas especies de mariposas, o cuando oía cantar los primeros ruiseñores, cucos y codornices"52. En esta reflexión sobre la $\mathrm{Cl}$, comparando un médico consigo mismo -"receptores de uno mismo"- a lo largo del tiempo ${ }^{53}$, creemos que se introducen matices "realistas". Este método se encuentra cercano a otros cualitativos y narrativos, alguno de los cuales se utilizan como parte de la formación médica ${ }^{54}$.

En resumen, observamos que la $\mathrm{Cl}$ es difícil, y que ha devenido en un escenario de demanda creciente cada vez más trivial, por parte de la población que desea lograr las pseudoventajas que se le ofrecen a través del consumo de pseudoservicios sanitarios. Hay una falta de comprensión por parte del paciente de lo que constituye una buena calidad científicotécnica por parte del médico, y una comunicación con el médico inapropiada y querulante en la forma por parte del paciente, y una defensa cognitiva y conductual del médico ante las pretensiones hipertrofiadas de los pacientes. En este contexto, podrían describirse distintas tipologías de $\mathrm{Cl}$, que pueden combinarse a lo largo del tiempo, y que podría ser discontinua y experimentarse como "momentos", presentando efectos positivos, pero también negativos (la $\mathrm{Cl}$, como el uso continuo de un fármaco -"el médico medicamento" de Balint ${ }^{55}$-, podría a la larga neutralizar su efecto benéfico; en ocasiones, desplazar la atención hacía otro médico puede ser de utilidad). Estos "resultados" solo pueden ser conjeturales, sujetos a argumentaciones a favor y en contra, materia para la investigación, las hipótesis, la dialéctica. Pretendemos, como Piranesi en sus grabados, mostrar perspectivas que hagan que realidades conocidas revelen algo nuevo.

De nuestra reflexión surgen muchas líneas y preguntas de investigación. ¿Cuál es el tiempo medio que un médico permanece en la misma consulta? ¿Cómo afecta la permanencia de un mismo médico en una misma consulta a la dinámica asistencial? ¿Qué factores explican la mayor o menor rotación de médicos? ¿Qué puede hacerse para estimular la permanencia (si es que se quiere estimular)? ¿Cómo examinar con precisión los resultados relacionados con la $\mathrm{Cl}$ y realizar mediciones reales de la variable que se está tratando de estudiar? ¿Es la conexión personal inherente a la $\mathrm{Cl}$ un elemento esencial? ¿Si esta $\mathrm{Cl}$ se erosiona aún más en el futuro, se verá afectada la esencia de la relación de curación? ¿ La Cl es la esencia de la MF, pero también su perdición? Sería preciso un estudio con decenas de "capturas de la relación" de médicos con sus pacientes a lo largo del tiempo, para aproximarnos algo más a esta cuarta dimensión de la asistencia médica, el tiempo. Pero, como decía Kaváfis: "Y siendo ya tan viejo, con tanta experiencia, sin duda sabrás ya qué significan las Ítacas" ${ }^{56}$.

\section{BIBLIOGRAFÍA}

1. Beaulieu MD. Teaching the essence of family medicine. Can Fam Physician. 2013;59(9):1017. Disponible en: http://www.ncbi.nlm.nih.gov/pmc/articles/PMC3771732/ [consultado 25 Abril 2015].

2. Stokes T, Tarrant C, Mainous III AG, Schers H, Freeman G, Baker R. Continuity of Care: Is the Personal Doctor Still Important? A Survey of General Practitioners and Family Physicians in England and Wales, the United States, and the Netherlands. Ann Fam Med. 2005;3(4):353-9. Disponible en: http://www.annfammed.org/content/3/4/353.full [consultado 25 Abril 2015].

3. Haggerty J, Burge F, Lévesque JF, Gass D, Pineault R, Beaulieu MD, et al. Operational Definitions of Attributes of Primary Health Care: Consensus Among Canadian Experts. Ann Fam Med. 2007;5(4):336-44. Disponible en: http://www.annfammed.org/cgi/content/full/5/4/336 [consultado 25 Abril 2015].

4. Cammer A, Morgan D, Stewart N, McGilton K, RycroftMalone J, Dopson S, et al. The Hidden Complexity of Long-Term Care: How Context Mediates Knowledge Translation and Use of Best Practices. Gerontologist. 2014;54(6):1013-23. Disponible en: http://gerontologist. oxfordjournals.org/content/54/6/1013.full [consultado 25 Abril 2015].

5. Haggerty JL, Reid RJ, Freeman GK, Starfield BH, Adair CE, McKendry R: Continuity of care: a multidisciplinary review. BMJ. 2003;327:1219-21. Disponible en: http://www. ncbi.nlm.nih.gov/pmc/articles/PMC274066/ [consultado 25 Abril 2015].

6. Williamson AE, Mullen K, Wilson P. Understanding "revolving door" patients in general practice: a qualitative study. BMC Fam Pract. 2014;15:33. Disponible en: http://www. biomedcentral.com/1471-2296/15/33 [consultado 25 Abril 2015].

7. Saultz JW. Defining and Measuring Interpersonal Continuity of Care. Ann Fam Med. 2003;1(3):134-43. Disponible en: http://www.annfammed.org/content/1/3/134.full [consultado 25 Abril 2015].

8. Murray SA, Kendall M, Boyd K, Sheikh A. Illness trajectories and palliative care. BMJ. 2005;330:1007. Disponible en: http://bmj.com/cgi/content/full/330/7498/1007?ecoll [consultado 25 Abril 2015]. 
9. Roger Garzón F. La relación médico-enfermo en el cuadro de las relaciones interpersonales. Comunicación presentada en la $17^{\text {a }}$ Semana de Ética y Filosofía. Congreso Internacional de la Asociación Española de Ética y Filosofía Política (AEEFP). Donostia-San Sebastián, 1-2-3 de Junio de 2011. Disponible en: http://www.uv.es/gibuv/Roger/ MedicoenfRoger.pdf [consultado 25 Abril 2015].

10. Ruiz Moral R, Rodríguez JJ, Epstein R. ¿Qué estilo de consulta debería emplear con mis pacientes?: reflexiones prácticas sobre la relación médico-paciente. Aten Primaria. 2003;32(10):594-602. Disponible en: http://www. elsevier.es/es-revista-atencion-primaria-27-articulo-queestilo-consulta-deberia-emplear-13055445 [consultado 25 Abril 2015].

11. Kondro W. Medical errors increasing because of complexity of care and breakdown in doctor-patient relationship, physician consultant says. CMAJ. 2010;182 (13):6456. Disponible en: http://www.cmaj.ca/cgi/content/ full/182/13/E645?etoc [consultado 25 Abril 2015].

12. Phan K; Brown SR. Decreased continuity in a residency clinic: a consequence of open access scheduling. Fam Med. 2009;41(1):46-50. Disponible en: http://www.kfinder. $\mathrm{com} / \mathrm{member}$-search/getdoc.cgi?data $=6 \mathrm{LOfZkDd8uNM} .8$ rGXu28DM6SZ8m0hw389JXR884GHv68.nGpZ8sUhQT8ChFQY82WxtA8DOaOO8IQHPg8xb5u7826xsM8QGpwr8vhIXtdB7CXPdL6\&grp=MDCHOICE\&z [consultado 25 Abril 2015].

13. Ridd MJ, Lewis G, Peters TJ, Salisbury C. Patient-Doctor Depth-of-Relationship Scale: Development and Validation. Ann Fam Med. 2011;9(6):538-45. Disponible en: http:// www.annfammed.org/content/9/6/538.full [consultado 25 Abril 2015]

14. Tarrant C, Windridge K, Baker R, Freeman G, Boulton M. Falling through gaps': primary care patients' accounts of breakdowns in experienced continuity of care. Fam Pract. 2015;32(1):82-7. Disponible en: http://fampra.oxfordjournals.org/content/32/1/82.abstract?etoc. [consultado 25 Abril 2015].

15. Kristjansson E, Hogg W, Dahrouge S, Tuna M, MayoBruinsma L, Gebremichael G. Predictors of relational continuity in primary care: patient, provider and practice factors. BMC Fam Pract. 2013;14:72. Disponible en: http://www.biomedcentral.com/1471-2296/14/72 [consultado 25 Abril 2015].

16. Hjortdahl P, Borchgrevink CF. Continuity of care: influence of general practitioners' knowledge about their patients on use of resources in consultations. BMJ. 1991;303:1181-4 Disponible en: http://www.ncbi.nlm.nih.gov/pmc/articles/ PMC1671517/ [consultado 25 Abril 2015].

17. Freeman GK. Progress with relationship continuity 2012 , a British perspective. Int J Integr Care. 2012;12: e128. Disponible en: http://www.ncbi.nlm.nih.gov/pmc/articles/ PMC3440251/ [consultado 25 Abril 2015].

18. Gervás J, Pérez Fernández M, Sánchez Sánchez RJ. Longitudinalidad, prestigio, buena reputación (social y profesional) y medicina general/de familia. Aspectos clínicos y de salud pública. Informe SESPAS 2012. Gac Sanit .2012;26 Supl 1: 52-6. Disponible en: http://gacetasanitaria.org/es/longitudinalidad-prestigio-buena-reputacionsocial/articulo/S0213911111003360/ [consultado 25 Abril
2015].

19. Beadles CA, Voils CI, Crowley MJ, Farley JF, Maciejewski $\mathrm{ML}$. Continuity of medication management and continuity of care: Conceptual and operational considerations. SAGE Open Med. 2014;2:1-7. Disponible en: http://smo.sagepub.com/content/2/2050312114559261.full [consultado 25 Abril 2015].

20. Atlas SJ, Grant RW, Ferris TG, Chang Y, Barry MJ. Patient-Physician Connectedness and Quality of Primary Care. Ann Intern Med. 2009; 150(5): 325-35. Disponible en: http://www.ncbi.nlm.nih.gov/pmc/articles/ PMC2975389/ [consultado 25 Abril 2015].

21. Cabana MD, Jee SH. Does continuity of care improve patient outcomes? J Fam Pract. 2004;53(12):974-80. Disponible en: http://www.ncbi.nlm.nih.gov/pubmed/15581440 [consultado 25 Abril 2015].

22. Kmietowicz Z. Seeing the same GP each time can reduce emergency department attendance, review finds. BMJ. 2014;349:g4847. Disponible en: http://www.bmj.com/ content/349/bmj.g4847? etoc [consultado 25 Abril 2015].

23. Campbell SM, Kontopantelis E, Reeves D, Valderas JM, Gaehl E, Small N, et al. Changes in Patient Experiences of Primary Care During Health Service Reforms in England Between 2003 and 2007. Ann Fam Med. 2010;8(6):499506. Disponible en: http://www.annfammed.org/content/8/6/499.full [consultado 25 Abril 2015].

24. Ackerman D. Una historia natural de los sentidos. Barcelona: Anagrama S.A.; 1992.

25. Martinez López de Letona J. La historia natural de la enfermedad como fuente esencial para la formulación de pronóstico. Monografías del Grupo Hospital de Madrid. Madrid: Editorial Materia; 2008.

26. Morrell D. The art of general practice. Oxford: Oxford University Press; 1991.

27. Woo B. Primary Care - The Best Job in Medicine? N Engl J Med. 2006;355:864-6. Disponible en: http://content.nejm.org/cgi/content/full/355/9/864 [consultado 25 Abril 2015].

28. Bigelow J. A discourse on self-limited diseases, delivered before the Massachusetts Medical Society at their annual meeting, May 27, 1835. Boston: Nathan Hale. Disponible en: http://archive.org/stream/natureindisease03bigegoog/natureindisease03bigegoog_djvu.txt [consultado 25 Abril 2015].

29. Haggerty JL, Roberge D, Freeman GK, Beaulieu C. Experienced Continuity of Care When Patients See Multiple Clinicians: A Qualitative Metasummary. Ann Fam Med. 2013;11(3):262-71. Disponible en: http://www.annfammed.org/content/11/3/262.full [consultado 25 Abril 2015].

30. Santucci A. Il colore del tempo. Muri, intonaci e mattoni della Bologna antica e di quella presente. Bologna: Centro Editoriale S. Stefano s.r.l.; 2000.

31. Claude Monet - La serie de la Catedral de Rouen, 18921894 - El clímax del impresionismo. Theartwolf.com. [homepage on the Internet] http://www.theartwolf.com/ monet_cathedral_es.htm 
32. Olesen F, Dickinson J, Hjortdahl P. General practice-time for a new definition. BMJ. 200; 20:326-7. Disponible en: http://www.bmj.com/content/320/7231/354.full [consultado 25 Abril 2015].

33. Turner J, Pugh J, Budiani D. "It's Always Continuing": First-Year Medical Students' Perspectives on Chronic IIIness and the Care of Chronically III Patients. Acad Med 2005; 80(2):183-8. Disponible en: http://www.ncbi.nlm. nih.gov/pubmed/15671326 [consultado 25 Abril 2015].

34. Jovell AJ. La historia natural de la profesión médica vista por un paciente. [homepage on the Internet]. http://www. fundacionmhm.org/pdf/Mono7/Articulos/articulo3.pdf

35. Koeck C. Imbalance of power between patients and doctors. BMJ. 2014;349:g7485. Disponible en: http://www. bmj.com/content $/ 349 / \mathrm{bmj} . \mathrm{g} 7485$ ? etoc $=$ [consultado 25 Abril 2015].

36. Bloom P. The Baby in the Well. The case against empathy. The New Yorker 2013, May 20. [homepage on the Internet]. http://www.newyorker.com/magazine/2013/05/20/the-baby-in-the-well

37. Arora NK, McHorney CA: Patient preferences for medical decision making: who really wants to participate? Med Care. 2000;38:335-41. Disponible en: http://journals.Iww. $\mathrm{com} / \mathrm{lww}$-medicalcare/pages/articleviewer.aspx?year $=200$ 0\&issue $=03000 \&$ article $=00010 \&$ type $=$ abstract [consultado 25 Abril 2015].

38. Tudor Hart J. Democratise or Perish: The Health Sciences as a Path for Social Change. En: Necessary and unnecessary utopias. Socialist Register 2000. Leo Panitch and Colin Leys (Editors). Suffolk: Merlin Press Ltd; 1999. Disponible en: http://socialistregister.com/index.php/srv/ article/view/5742 [consultado 25 Abril 2015].

39. El sol del membrillo. Wikipedia. [homepage on the Internet]. http://es.wikipedia.org/wiki/El_sol_del_membrillo

40. Leal Hernández $M$, Abellán Alemán J, Gómez Jara $P$, Martín-Sacristán Martín B. ¿Por qué se cambian de médico nuestros pacientes? ¿Qué opinamos los médicos de familia? Aten Primaria. 2007;39(10):576. Disponible en: http://www.elsevier.es/es-revista-atencion-primaria27-articulo-por-que-se-cambian-medico-nuestros-pacientes-13110741 [consultado 25 Abril 2015]

41. Mercer SW, Guthrie B, Furler J, Watt GCM, Tudor Hart J. Multimorbidity and the inverse care law in primary care. BMJ. 2012;344:e4152. Disponible en: http://www.bmj. com/content/344/bmj.e4152?etoc [consultado 26 Abril 2015].

42. Reyre A, Jeannin R, Larguèche $M$, Hirsch $E$, Baubet $T$, Moro MR, et al. Care and prejudice: moving beyond mistrust in the care relationship with addicted patients. Medicine, Health Care and Philosophy. 2014;17(2):18390. Disponible en: http://alerts.springer.com/ re?l=DOIn5qbl7l6hdyl6kl5 [consultado 26 Abril 2015].

43. Gandhi IG, Parle JV, Greenfield SM, Gould S. A qualitative investigation into why patients change their GPs. Fam Pract. 1997;14(1):49-57. Disponible en: http://www.ncbi. nlm.nih.gov/pubmed/9061345 [consultado 26 Abril 2015].

44. Spinewine A, Claeys C, Foulon V, Chevalier P. Approaches for improving continuity of care in medication management: a systematic review. Int J Qual Health Care. 2013;25(4):403-17. Disponible en: http://intqhc.oxfordjournals.org/content/25/4/403.long [consultado 26 Abril 2015].

45. Turabián JL, Pérez Franco B. Reflexiones sobre el presente y el futuro de la medicina de familia. Gac Sanit. 2014;28:259. Disponible en: http://www.gacetasanitaria.org/es/reflexiones-sobre-el-presente-el/articulo/ S0213911113001660/ [consultado 26 Abril 2015].

46. Starfield B. Atención primaria. Equilibrio entre necesidades de salud, servicios y tecnología. Barcelona: Masson; 2001.

47. Spence D. Continuity is never out of fashion. BMJ. 2012;344:e957. Disponible en: http://www.bmj.com/content/344/bmj.e957? etoc= [consultado 26 Abril 2015].

48. Confucio. Los cuatro libros clásicos. Barcelona: Editorial Bruguera S. A.; 1974.

49. Mathews A, Steptoe A. Essential Psychology for medical practice. Edinburgh: Churchill livingstone, 1988.

50. Cox C, Mead A (Ed.). A sociology of medical practice. London: Collier-MacMillan; 1975.

51. Cruz Martínez O, Fragoso Marchante MC, González Morales I, Sierra Martínez D P, Labrada González JA. La relación médico paciente en la actualidad y el valor del método clínico. MediSur. 2010;8(5):110-20. Disponible en: http://www.redalyc.org/pdf/1800/180020098021.pdf [consultado 26 Abril 2015].

52. Delibes M, Delibes de Castro M. La tierra herida. ¿Qué mundo heredaran nuestros hijos? Barcelona: Ediciones Destino; 2007.

53. Roth W-M. Auto/biography as Method: Dialectical Sociology of Everyday Life. Review Essay: Zdenek Konopásek (Ed.) (2000). Our Lives as Database-Doing a Sociology of Ourselves: Czech Social Transitions in Autobiographical Research Dialogue. Forum Qual Soc Res. 2002;3(4):17. Disponible en: http://www.qualitative-research.net/fqstexte/4-02/4-02review-roth-e.htm [consultado 26 Abril 2015].

54. Ogur B, Hirsh D. Learning through longitudinal patient care-narratives from the Harvard Medical School-Cambridge Integrated Clerkship. Acad Med. 2009;84(7):844-50. Disponible en: http://journals.Iww.com/academicmedicine/ pages/articleviewer.aspx year $=2009 \&$ issue $=07000 \& a r t i c l e$ $=00011 \&$ type $=$ abstract [consultado 26 Abril 2015] .

55. Balint E, Norell JS (Ed). Six minutes for the patient: interactions in general practice consultations. London: Tavistock publications; 1973.

56. Cavafis CP. Antología poética. Madrid: Alianza Editorial; 1999. 